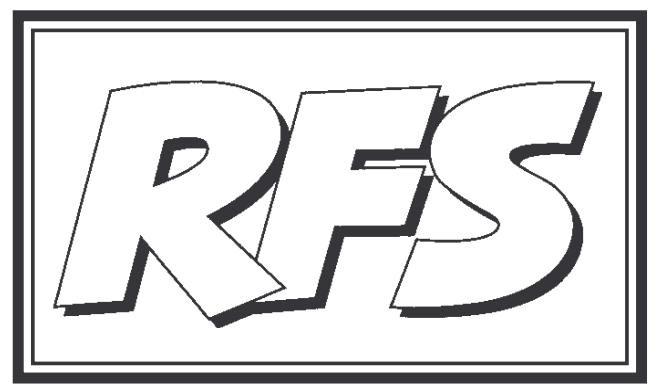

Revista de Fomento Social, 61 (2006), 603-630

\title{
Los compromisos de la cooperación internacional. Balance de los Objetivos de Desarrollo del Milenio en la India
}

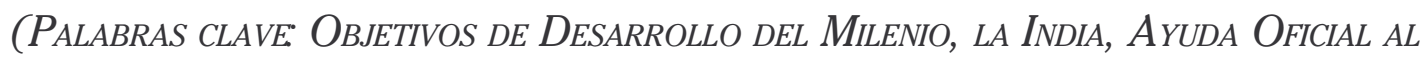
Desarrollo (AOD), CoOperaCión MULTILATERAL, COOPERACIÓN BILATERAL, ONGD.

KEY WORDS: MILLENNIUM DEVELOPMENT GOALS, INDIA, OFFICIAL AID FOR DEVELOPMENT (OAD), Multilateral COOPERATION, BILATERAL COOPERATION, NEO)

${ }^{1}$ Licenciada en Sociología. Master en Cooperación Internacional y Gestión de Proyectos de Desarrollo. Mis más sinceros agradecimientos a Antonio García Lizana, quien me ha pres tado su ayuda, tanto en este artículo, como a lo largo de mi trayectoria como técnico de desarrollo. 


\section{Introducción}

Desde hace décadas, la lucha contra la pobreza ha venido siendo cuestión central para las diferentes agencias nacionales e internacionales del panorama de la Cooperación al Desarrollo.

Una de las más importantes cumbres fue la realizada en el año 2000 por la ONU (Organización de Naciones Unidas), donde 191 países adoptaron la declaración del milenio, a partir de la cual se han definido los 8 Objetivos de Desarrollo del Milenio (ODM), insertos en las teorías del Desarrollo Humano, como marco para conducir la Cooperación Internacional, dándose un plazo de 15 años para la consecución de dichos objetivos. Por primera vez en la historia de la cooperación al desarrollo, la ayuda tenia un camino común que seguir. Desde entonces, el mundo está avanzando hacia la consecución de los ODM, pero este progreso es desigual y demasiado lento.

Cada ODM se compone por una o varias metas, seguidas de diferentes indicadores o medidores de desarrollo, que facilitan la concreción y la tarea de evaluación. Los 8 ODM son los siguientes:

Objetivo 1. Erradicar la pobreza extrema y el hambre.

Objetivo 2. Lograr la enseñanza primaria universal.

Objetivo 3. Promover la igualdad entre los géneros y la autonomía de la mujer.

Objetivo 4. Reducir la mortalidad de los niños menores de 5 años.

Objetivo 5. Mejorar la salud materna.

Objetivo 6. Combatir el VIH/SIDA, malaria y otras enfermedades.

Objetivo 7. Garantizar la sostenibilidad del medio ambiente.

Objetivo 8. Crear de una alianza global de desarrollo.

Según el Informe del CAD (2005), el objetivo de reducción de pobreza es el único que presenta un avance a nivel global. Desde el punto de vista regional, el Informe señala que África Subsahariana no está bien situada para alcanzar ningún objetivo. El Magreb y Oriente Medio progresan ligeramente en los objetivos sociales. El Sur de Asia encuentra sus mayores dificultades en los objetivos de salud, educación e igualdad de género. En Extremo Oriente y el Pacífico, es la salud infantil la que peor situada se encuentra. Por último, Asia Central, Europa del Este, Iberoamérica y el Caribe reducen su pobreza pero de forma lenta. 
El objetivo perseguido en el presente artículo es centrarnos en la situación existente en un solo país, la India, cuyas características lo convierten, desde todos los puntos de vista, en un caso paradigmático.

\section{Marco contextual ${ }^{2}$}

Bharat Juktarashtra o República de la India, es un país con 1.064 millones de personas, con una esperanza de vida de 63 años, de las que el $29 \%$ vive en o por debajo del umbral de pobreza nacional. El 90\% de la pobreza se aglomera en 12 de los 28 Estados de la India, siendo estos: Andhra Pradesh, Assam, Jharkjand, Karnataka, Maharashtra, Orissa, Rajasthan, TamilNadu, Bengala Oriental, Uttar Pradesh, Bihar y Madhya Pradesh, donde se encuentran aproximadamente el $90 \%$ de los pobres, con una concentración que supera los 133 millones de pobres en los tres últimos. Tres de cada cuatro, viven en áreas rurales, el porcentaje de población total que vive en dicha área es un $72 \%$. La agricultura, junto al sector industrial son los que generan la mayor proporción del PIB (600,6 mil millones de $\$$, con un crecimiento anual de $8,6 \%)$. No obstante, las empresas de pequeño tamaño, de propiedad familiar basadas en la artesanía son las más importantes en cuanto a la generación de empleo. El índice de Gini es de 0,32 , lo que refleja la desigualdad sufrida y el Índice de Desarrollo Humano (2004) presenta un 0,59, colocando a la India en el puesto 127, sobre 175.

El sello distintivo de la cultura india es su gran variedad religiosa y lin güística. Su estratificación social se basa en 4 castas inamovibles. Fuera del orden social están los sin casta, conocidos también como parias o dalits. Aunque este sistema de castas fue abolido por la constitución de 1950, si gue presente de forma contundente en la vida social del país, y es fuente de discriminación y segregación entre la población.

A su vez, existen serios problemas de desigualdad de género. Si bien, actualmente la mujer en la India está mejorando de forma progresiva su estatus social; también es cierto que todavía coexisten procesos de marginación

${ }^{2}$ Los datos se han obtenido de diversos informes que se encuentran en bibliografía. El año de referencia es el 2003, en los casos en que éste varia, es indicado. Hay que tener en cuenta la modificación de los datos, ante los hechos ocurridos en diciembre de 2004. Se estima que a causa del tsunami 10.749 personas perdieron sus vidas, 157.393 casas fueron destruidas y 897 villas se vieron afectadas, siendo Tamil Nadu el estado más afectado. Las cifras oficiales sobre población total tras lo ocurrido, todavía no son públicas. 
donde la mujer es considerada ciudadana de derechos y deberes, por lo que se convierte en agente pasivo del desarrollo de su propia comunidad. La desventaja comienza antes de nacer, la desvinculación familiar de la mujer (sociedad patrilocal) y el coste de reunir la dote tiene como consecuencia la generalización del feticidio. En su vida adulta, su situación de subordinación propiciará la gran violencia que las mujeres soportan, y la poca accesibilidad a los recursos, en especial los sanitarios, como los tratamientos farmacoló gicos. Síntoma también de este status que ocupa la mujer en esta sociedad patriarcal es su poco peso en la toma de decisiones.

En cuanto a la sanidad se han conseguido grandes mejoras desde su independencia (1947) hasta nuestros días; entre otras, se ha doblado la esperanza de vida, la malaria ha sido puesta bajo control y la viruela ha sido eliminada. Sin embargo, aun quedan graves asuntos por resolver. Existen entre 3,8 y 4,6 millones de personas seropositivas, de las que 600.000 necesitan tratamiento con urgencia. La tasa de mortalidad infantil y de mujeres continua siendo excesivamente elevada, un tercio del total de muertes ocurre en niños menores de 5 años y mujeres. Aunque se ha aumentado de forma considerable la tasa de población con acceso al agua potable, aún continua siendo uno de los países con menor accesibilidad. Además, menos de la quinta parte de la población rural tiene letrinas o servicios de saneamiento.

En educación también se han producido grandes avances desde su independencia, la tasa de matriculación en primaria es del 99\%, y el 95\% de la población rural ya tiene acceso a una escuela primaria dentro de un kilómetro de su casa. Aunque la tasa de alfabetización también ha aumen tando, todavía el problema es grave, ya que, en términos absolutos, casi 300 millones de personas mayores de 6 años continúan siendo analfabetas. Además, existe bastante disparidad entre zonas urbanas y rurales (con tasas de $80 \%$ y $59 \%$ a favor de los centros urbanos), entre los Estados, y según la casta de procedencia.

\section{Políticas de desarrollo internas}

El gobierno de la India elaboró en 2001 el 10 Plan Quinquenal (2002-2007), el cual constituye el plan director para el desarrollo y el crecimiento del país en ese período de tiempo. Así como también sirve de guía para la Ayuda Oficial al Desarrollo (AOD) internacional. Se compone de cuatro temas: 
Crecimiento económico. Es la columna vertebral del plan, que tiene como meta principal lograr una tasa de crecimiento del 8\% anual. Para lograrlo se tendrá que avanzar en temas como la reducción del déficit fiscal y de la deuda pública, además de un importante avance en las infraestructuras de desarrollo, en sectores como transporte y energía, con la finalidad de conectar las áreas más pobres con las ciudades más desarrolladas.

Desarrollo equitativo y justicia social.Se centra a su vez en tres vertientes: un rápido crecimiento de la agricultura debido al impacto que tiene sobre la población más pobre, creación de nuevos puestos de trabajo e implementa ción de nuevos programas destinados a los grupos menos privilegiados. En relación al primero, lo que se pretende es cambiar el esquema de seguridad alimentaría por productividad, para lo cual son necesarias ciertas reformas, como el cambio de los subsidios por inversión en infraestructuras rurales e incentivos para diversificar la producción.

Desarrollo Humano. Gira en torno a la educación y la salud. El gobierno es consciente de que en los últimos años se ha hecho un gran esfuerzo para elevar la tasa de matriculación escolar, por lo cual una de las prioridades se basa actualmente en la mejora de la calidad de la educación. Igualmente ésta debe ser ampliada a las castas menos privilegiadas que son menos pro pensas a enviar a sus menores a las instituciones educativas. Con relación a la salud, se pretende no sólo mejorar los servicios de salud pública sino también ampliarlos para que toda la población, en especial la más desfavorecida, tenga accesos a servicios de sanidad de alta calidad.

Reformas y Buena Gobernabilidad. Es la piedra angular del Plan. Establece que una gobernabilidad débil se manifiesta a sí misma en la impotencia para ofrecer servicios públicos de calidad, en regulaciones excesivas y en un incremento del gasto publico improductivo, constituyendo así las prin cipales trabas para el desarrollo. Para poder hacer frente a ello, establece la necesidad de mejorar: la transparencia, la responsabilidad, eficiencia y sensibilización de la administración pública a todos los niveles.

Como se puede comprobar, las políticas de desarrollo promovidas por el gobierno de la India van encaminadas a trabajar en temas comprendidos por los ODM; pese a que no se señalan ni las mismas metas ni, lógicamente, los mismos plazos, los objetivos marcados en el Plan guardan una estrecha relación con los ODM. De este modo, El ODM de "Erradicar la pobreza ex trema y el hambre", se ve reflejado en el plan bajo el propósito de reducir al $20 \%$ la población que vive bajo el umbral de la pobreza. El ODM de "Educa- 
ción primaria universal" mantendría la misma intención de resultados en la escolaridad de los menores. En correspondencia con el ODM de "Promover la igualdad entre los géneros y la autonomía de la mujer", no se dan datos concretos; sin embargo, existe una mejora en la sensibilización hacia este tema, destacándose como un sector prioritario e independiente de otros y poniendo énfasis en su "empoderamiento", en lugar de simplemente su "bien estar." En cuanto a los ODM de "Reducir la mortalidad de los niños menores de 5 años", "Mejorar la salud materna" y "Combatir el VIH/SIDA, malaria y otras enfermedades", se ven contemplados en el plan bajo los objetivos de sanidad: reducir la mortalidad infantil en un 45 por mil y la mortalidad materno infantil al 2 por mil para el 2007; así como, combatir VIH/SIDA, malaria, lepra, tuberculosis y otras enfermedades. En relación al ODM de "Garantizar la sostenibilidad del medio ambiente", el plan mantiene el objetivo de que todos los pueblos tengan acceso a agua potable para el 2007.

\section{La Ayuda Oficial al Desarrollo (AOD) en la India (2003-2004)}

La AOD ${ }^{3}$ percibida por la India (ver cuadro 1) entre 2001 y 2003 ha experi mentado un crecimiento sostenido, pasando de 477 millones de $\$$ a 2.455 . Los principales donantes (ver cuadro 3) han sido la AID (Agencia Internacional de Desarrollo), perteneciente al BM (Banco Mundial), seguido del JBIC (Banco de Cooperación Internacional del Japón), entre ellos existe una diferencia en la ayuda desembolsada de 137 millones de US\$; sin embargo, la mayor reducción de cuantía se encuentra entre este último y el siguiente donante del ranking, el DFID (Departamento de Desarrollo Internacional) del Reino Unido, la diferencia entre ellos es de 422 millones de US\$. Por sectores (ver gráfico 1), conviene advertir que la mayor concentración de recursos se produce en Infraestructura Económica y Servicios, seguido del sector de atribución no especificada, Salud y Población y Otros sectores sociales, los 3 con un porcentaje similar del 12\% aproximadamente.

${ }^{3}$ Las cifras oficiales sobre AOD percibida tras el tsunami ocurrido en diciembre de 2004, todavía no son públicas; así que, aún conscientes que éstas cifras pueden haber variado, trabajaremos con las aportadas hasta el momento, mayoritariamente con una máxima ac tualización de hace 3 años. 


\section{CUADRO 1. AOD percibida por la India}

\begin{tabular}{|l|c|c|c|}
\hline Ingresos & 2001 & 2002 & 2003 \\
\hline AOD Neta (millones de US\$) & 1.724 & 1463 & 942 \\
\hline \% de AOD Bilateral & $55 \%$ & $60 \%$ & $60 \%$ \\
\hline AOD Neta / PIB & $0.4 \%$ & $0.3 \%$ & $0.2 \%$ \\
\hline Flujo Neto de Capital Privado (millones) & 477 & 1.070 & 2.455 \\
\hline
\end{tabular}

Fuente: Organización para la Cooperación y el Desarrollo Económico (OCDE). Traducción propia.

\section{CUADRO 2. Otros datos relevantes}

\begin{tabular}{|l|c|c|c|}
\hline Referencias & 2001 & 2002 & 2003 \\
\hline Población (millones) & $1.032,5$ & $1.048,6$ & $1.064,4$ \\
\hline PIB per capita (Atlas USD) & 480 & 470 & 530 \\
\hline
\end{tabular}

Fuente: Organización para la Cooperación y el Desarrollo Económico (OCDE). Traducción propia.

\section{CUADRO 3. Principales donantes}

\begin{tabular}{|c|l|c|}
\hline \multicolumn{2}{|c|}{ Ranking de donantes de AOD Neta (2002-03) } & US\$ mm \\
\hline 1 & Agencia Internacional de Desarrollo & 905 \\
\hline 2 & Japón & 768 \\
\hline 3 & Reino Unido & 346 \\
\hline 4 & Alemania & 159 \\
\hline 5 & Estados Unidos & 149 \\
\hline 6 & Holanda & 105 \\
\hline 7 & CE & 79 \\
\hline 8 & Canadá & 31 \\
\hline 9 & UNICEF & 28 \\
\hline 10 & Suecia & 24 \\
\hline
\end{tabular}

Fuente: OCDE. Traducción propia. 


\section{GRÁFICO 1. AOD por sectores (2002-2003)}

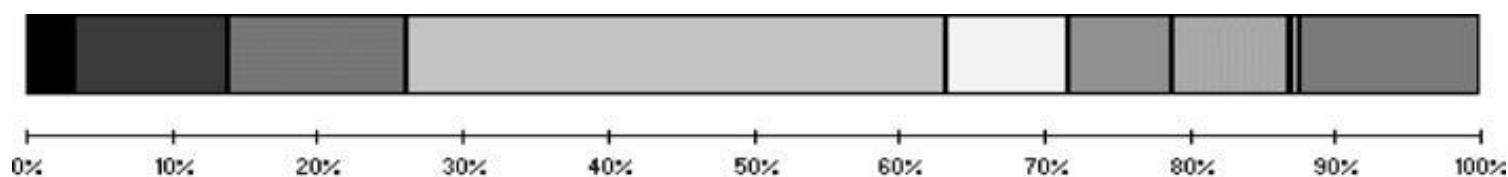

\section{Educación \\ Infraestructura Económica \& Servicios Programas de Asistencia Otros y No Especificados}

Salud \& Población Producción Deuda
Otros sectores sociales Multisectorial Asistencia de Emergencia

Fuente: OCDE. Traducción propia.

Con el propósito de tener una visión más precisa de la problemática planteada, procedemos a continuación a considerar la labor realizada por los donantes más significativos.

\subsection{Cooperación multilateral}

- El Banco Mundial (BM)

El BM apoya el desarrollo de la India a través de los diferentes programas y proyectos que se ejecutan en los diversos Estados del país, sobre todo en aquellos 12 que concentran el 90\% de los pobres. No obstante, se podrían señalar 4 estados donde el BM ha venido trabajando más activamente: Bihar, Jharkhand, Orissa, y Uttar Pradesh.

La actuación del Banco conjuntamente con el gobierno de la India, conlleva al desarrollo de un plan denominado CAS (Estrategia de Ayuda al País), que apoya el propio programa de desarrollo del Gobierno que es quien realiza sus propios proyectos con el financiamiento del BM y la ayuda técnica. Esta estrategia, que fue fijada en agosto de 2004, es y será usada para guiar los programas del BM en la India durante los próximos años (2005-2008). Se enfoca en tres áreas principales: a) Infraestructura: caminos, transporte, suministro de agua e higienización, irrigación y desarrollo urbano, b) Desarrollo Humano: educación, salud y protección social; x) Sustentos Rurales: con un énfasis en los acercamientos a las comunidades.

Los préstamos se realizan a través del propio BIRD (Banco Internacional de Reconstrucción y Desarrollo), donde existe un tipo de interés sobre los 
prestamos realizados (del 1,59\% a marzo 2004), así como, a través de la AID (Asociación Internacional para el Desarrollo), como parte del grupo del BM, que concede préstamos que no tienen ninguna carga o interés, pero sí una comisión por operación del $0,75 \%$ sobre la parte desembolsada del crédito. Este último es el principal donante de AOD destinada a la India.

De esta manera, los ODM a los que contribuye serán principalmente: erra dicación de la pobreza extrema y el hambre, lograr la enseñanza primaria universal, aquellos relacionados con la sanidad (objetivos 4, 5 y 6 ) y en menor medida, al ODM de garantizar la sostenibilidad del medio ambiente.

\section{- Banco Asiático de Desarrollo (BAD)}

La India es el cuarto socio más importante del BAD después de Japón, EE.UU. y China. A pesar de que fue uno de los países fundadores del BAD en 1966, el Gobierno de la India decidió aprovechar los préstamos del banco por primera vez en 1986, recibiendo hasta el año 2002 un total de 78 présta mos o unos 13 billones de dólares, así como unos 102 millones en asistencia técnica hasta 2003.

La política de cooperación del BAD para la India, hace hincapié sobre todo en la reducción de la pobreza a través del logro del crecimiento económico. Se trata de llevar a cabo el crecimiento teniendo en cuenta la importancia de promover y proteger al mismo tiempo la equidad social.

La intervención del BAD se implementa a través del "Documento de Estrategia País 2003-2006”. Se espera que en estos cuatro años el BAD desembolse recursos por 750 millones de US $\$, 850$ millones de US\$, 1.000 millones de US\$ y 1.200 millones de US\$, respectivamente. La estrategia está fundamentada en tres pilares: Desarrollo Pro-pobres, Desarrollo Social y Buena Gobernabilidad, siendo prioritario el primero. La agenda consistirá en dar soporte a los niveles más altos del gobierno con miras a consolidar la gestión fiscal, así como contribuir al desarrollo del sector privado y de la infraestructura para el desarrollo; además de la asistencia al desarrollo rural y a la agricultura, debido en gran medida a que estos sectores concentran el mayor número de pobres.

De hecho, durante 2003 la ayuda se enfocó en la mejora de las infraestruc turas económicas, adonde se canalizaron casi 2,075 millones de dólares, lo que supone el $89 \%$ de ayuda total percibida por la India en ese periodo. 
Por tanto, la mayor repercusión de la AOD desembolsada desde el BAD cae sobre el ODM de erradicación de la pobreza extrema y el hambre, con reper cusión también en el de garantizar la sostenibilidad del medio ambiente.

- Programas de Naciones Unidas para el Desarrollo (PNUD)

El PNUD ha apoyado el desarrollo de la India en las últimas cinco déca das. Esta institución hace un gran esfuerzo para realizar su trabajo sobre la base de la teoría del desarrollo humano; para ello ha creado en el país un Centro de Recursos de Desarrollo Humano, donde se recopila, analiza y pro cesa información como herramienta de trabajo, diferente a los indicadores económicos que utilizan otros organismos en la cooperación. El Programa para el País (2003-2007) se concentra en 5 estados: Uttar Pradesh, Andhra Pradesh, Orissa, Bihar y Punjab. El presupuesto destinado a este programa es de 200 millones de US\$. Los temas del Programa son :

Promover el desarrollo humano, la igualdad de género, y el desarrollo de la capacidad de descentralización. Se promoverá a través de dos vías: a) Fortalecimiento de los planes de desarrollo del gobierno, con los objetivos de asegurarse que el informe de desarrollo es parte de la agenda nacional y estatal, crear capacidad en los gobiernos locales (panchayats) para la eficaz provisión de los servicios y promover la participación ciudadana, la transparencia, el fortalecimiento del sistema estadístico e informático, e identificación de estrategias de financiación, y b) Promoción de la igualdad de género.

Desarrollo en zonas pobres rurales y urbanas . El programa comprende: a) Estrategia para los pobres de zonas urbanas: partiendo del estudio de la supervivencia de la gente en las ciudades se busca la mejora de su situación, a través de créditos y ayuda técnica, al mismo tiempo que se pretende hacer un plan más ambicioso donde se integren actividades artesanales, servicios intensivos en mano de obra y servicios de cooperativas, b) El turismo endógeno, el ecoturismo: es un proyecto de turismo alternativo que se entiende como una buena forma de crear empleo y proporcionar un medio de vida sostenible; tiene aplicación en 18 localidades del país y parte de la sensibilización y movilización de los grupos locales en esta materia, creando centros y museos de arte local y realizando cursos de formación en hostelería, con especial atención de la participación de las mujeres y grupos desfavorecidos, c) Energía renovable para áreas rurales: el uso de 
estas energías ha beneficiado a muchas familias rurales: se ha aliviado de trabajo pesado a muchas mujeres, se han reducido los riesgos en salud, se han creado oportunidades de empleo y se ha disminuido el avance de la deforestación. El objetivo es que estas comunidades gestionen y mantengan estos recursos energéticos. Se ha implementado en 60 pueblos pilotos, con especial atención a poblaciones indígenas, y d) Gestión de recursos naturales de la comunidad: el crecimiento de la población, el consumo insostenible, el empobrecimiento de los conocimientos tradicionales locales y la agricultura intensiva han llevado a una mayor degradación del medio ambiente, tanto en cantidad, como en calidad. Esto genera mayor vulnerabilidad de las comunidades rurales, especialmente de las mujeres, y extensión de la pobreza.

Apoyo para el desarrollo de una respuesta efectiva del problema del SIDA en la India. Se materializa en el proyecto CHARCA, destinado a mujeres jóvenes entre 13 y 25 años, con el objetivo de ayudar a la mujer a protegerse a sí misma y tomar conciencia de la situación, así como mejorar su acceso a los servicios de salud. Es un programa donde se pone a prueba la coordinación entre ONGs, gobiernos y ONU.

Se puede afirmar que los programas del PNUD abarcan la totalidad de los ODM, debiendo tomar en consideración en relación con el mismo el esfuerzo combinado de las distintas agencias especializadas de la $\mathrm{ONU}^{4}$ en los diferentes campos de su incumbencia.

4 Múltiples son las agencias pertenecientes a la ONU que trabajan activamente en la India. No obstante, destaca entre ellas UNICEF, ya que es la organización más grande de la ONU en el país. El programa Gobierno-UNICEF 2003-2007 tiene las siguientes prioridades: a) Reducir la mortalidad infantil y la mortalidad de la mujer en el parto, a través de interven ciones en sanidad, agua potable, higiene pública, nutrición, y con atención especial a las niñas y a las mujeres, b) Reducir la malnutrición infantil y el bajo peso al nacer, desarrollo adecuado del bebé y mejora de su cuidado, c) Asegurar la educación primaria para todos los niños, d) Aumentar la protección infantil incluyendo la eliminación progresiva del tra bajo infantil, el abuso sexual infantil y el tráfico de niños, y e) Prevenir VIH/SIDA entre los niños, adolescentes y familias. Además, se han creado grupos de trabajo entre las agencias para programas que requerían colaboración entre ellas. Trabajan conjuntamente en temas de explotación infantil, educación primaria, nutrición, sanidad, agua, género y desarrollo rural. 
- La Unión Europea (UE)

La cooperación entre la UE y la India se inició hace aproximadamente 30 años y actualmente se rige por el Acuerdo de Cooperación de $1994{ }^{5}$. En cuanto a la base jurídica de dicha cooperación se encuentra en el reglamento ALA. Podemos dividir la cooperación al desarrollo de la UE con la India en 4 ámbitos:

Educación. La UE mantiene 3 programas que comparten la finalidad de la universalización de la educación:a) Programa de educación primaria a nivel distrito (DPEP). Las zonas elegidas son las de tasas de pobreza mas altas y menor ratio de escolaridad infantil, poniendo especial énfasis en la escolaridad femenina. Se implanta actualmente en 275 distritos en 18 estados, b) Programa para garantizar la educación a los grupos más excluidos (EGS). Es una alternativa a las escuelas formales, su importancia radica en el hecho de que une la dimensión de empoderamiento comunal con la de educación, especialmente en las áreas tribales donde la accesibilidad a las escuelas es escasa, yc) Programa para la mejora del nivel de Educación escolar (PESLE). Destinado a grupos especialmente vulnerables y marginados, se trabaja en pro de un desarrollo de "buenas practicas escolares".

Sanidad. La UE implementa los siguientes programas: a) Programa para la inversión en salud y bienestar familiar (SIP). El objetivo del programa se basa en la reforma del sistema de sanidad, focalizado esencialmente en los servicios de salud primaria, dotando de capacidad a las instituciones e incluyendo la participación social en ello, b) Lucha contra el VIH/SIDA, a través de un programa de apoyo presupuestario, trabajado por ONGs con un total de 90 proyectos, yc) Campaña contra el abuso de drogas, en la misma línea de apoyo presupuestario, se plantean iniciativas para evaluar los problemas de la drogadicción y se ayuda a la rehabilitación de personas con la adicción. Las intervenciones de la UE se basan en compartir su experiencia en la cooperación regional en base a este tema.

Desarrollo rural y recursos naturales. Contiene los siguientes programas: a) Rehabilitación del Medio Ambiente, a través de investigación en nuevas energías y la preocupación por el carácter de sostenibilidad ambiental de los proyectos implantados, $b$ ) Proyectos de recuperación de tierras. La India posee vastas áreas potencialmente cultivables que son perdidas debido a la

${ }_{5}^{5}$ Para ver el acuerdo: $\quad$ http://europa.eu.int/comm/external relations/india/intro/agree08 $\underline{94 . p d f}$ 
erosión física o a la degradación química, en este sentido el proyecto de la UE "Saline Lands Reclamation in Maharashtra, Phase II" se destina a implementar técnicas para la recuperación de tierras en dicha zona,c) Proyectos de silvicultura: (Haryana Community Forestry), este proyecto se construye a partir de las experiencias de trabajo con distintas instituciones locales en la gestión de los recursos para la reforestación, su objetivo es incrementar la calidad de vida de las comunidades rurales a través de un desarrollo sostenible. Estos proyectos se encuentran implantados actualmente en 3.000 zonas rurales, $d$ ) Proyectos para la integración de la línea divisoria de aguas: a través de 2 proyectos: 1 ) Doon Valley Project Ravine. El objetivo es contrarrestar la degradación que sufre el valle por la mano del hombre y causas naturales, la UE aporta un equipo de asistencia técnica. Al proyecto le sigue una estrategia de desarrollo grupal, ya que 250 villas son formadas para implementar micro-planes de irrigación, conservación de suelo y agua y actividades de conservación de energía, 2) Ravine Stabilisation in Uttar Pradesh. Busca la prevención de la erosión del suelo a través de la conservación de tierras, reforestación, horticultura y ganadería, está implantado en 75 villas, $e$ ) Proyectos de irrigación. Se trata de la construcción de canales de irrigación y formación para el mantenimiento de estos. Actualmente se llevan a cabo 3 proyectos: 1) Proyecto de Irrigación en Sidhmukh y Nohar, 2) Proyecto de irrigación menor en Orissa y 3) Proyecto de rehabilitación de tanques en Pondicherry. También se trabaja en Tamil Nadu, Kerala y Rajasthan, y f) Producción y marketing del sector agricultura. Se trata de políticas encaminadas al desarrollo rural a través de iniciativas de mejoras en su producción, tratamiento y marketing, promoviendo empleo y oportur nidades para las zonas rurales y semiurbanas. El programa de desarrollo de horticultura en Kerala (KHDP), consiste en 8 proyectos piloto con el objetivo de incrementar los ingresos de los agricultores a través de iniciativas para incrementar su productividad. Para la mayor eficacia de éste se crearon los llamados grupos de autoayuda (SHG), a los que se otorgan créditos con facilidades, prácticas de formación y otros servicios.

Democracia y derechos humanos. Se basa en distintas iniciativas:a) Iniciativa europea por la democracia y los derechos humanos (EIDHR), dedicada a la prevención y rehabilitación de victimas de tortura, combatir el racismo y la xenofobia y proteger minorías indígenas, yb) Contrato con la ONG TOP (Centro de prevención de torturas), cuyo objetivo es cambiar la actitud de la gente hacia las victimas de torturas a través de la sensibilización.

Además, el gobierno de la India y la UE son conscientes de la importancia de 
aumentar el mutuo entendimiento, la movilización de canales de la sociedad civil ha sido vital para lograr este objetivo, en este sentido la UE mantiene un programa de cultura: EU-India Economic Cross Cultural Programme (ECCP), el cual contribuye a esta dedicación como clave para proteger el marco de unión entre la sociedad civil en India y la UE. Este programa fue lanzado en 1997, desde entonces se han llevado a cabo 60 proyectos, los proyectos firmados en el año 2004 son dos: a) "Circles of Support in India and the EU - Developing qualification modules for person-centred community inclusion networks with vulnerable individuals: A project in Germany, India, and Britain". Se trata de crear círculos de apoyo entre la India y la UE, con una duración de 24 meses, se basa concretamente en apoyar la igualdad de oportunidades, la participación económica y el empoderamiento de la gente con diferentes re cesidades yb) "Cultural Dimensions in Digital Multimedia Security Technology (CultureTech)". El principal objetivo es unir y programar el conocimiento de tecnologías, relacionado todo ello con asuntos interculturales.

Aunque, principalmente, la UE proporciona ayuda de forma sectorial para el logro de los ODM referidos a educación (objetivo 2) y sanidad (objetivos 4, 5 y 6), contribuye también, al ODM de garantizar la sostenibilidad del medio ambiente. Su aportación al objetivo de igualdad de género es tratando el tema de forma transversal en todos sus programas. Diferenciándose del resto de los donantes, la UE trabaja activamente en el ODM de crear una alianza global ${ }^{6}$ de desarrollo. Además, hay que señalar que es el único actor de cooperación que implementa en la India un programa de diálogo interreligioso o intercultural, desde la perspectiva del mutuo entendimiento, en pro de la mejora de las relaciones entre la población y con el objetivo de eliminar posibles conflictos. Éste último no es un ODM; sin embargo, dadas las características intrínsecas del país, se hace absolutamente necesario.

\subsection{Cooperación bilateral}

Durante años la India ha recibido ayuda bilateral de un gran número de países. Podemos contar en los diferentes presupuestos generales hasta 30 países que han otorgado préstamos o subvenciones a la India. Esa ayuda

$6 \quad$ Este objetivo consiste en establecer un ambiente entre los países donantes y receptores en el cual un desarrollo rápido y sostenible sea posible. Además, pretende crear una conciencia de responsabilidad compartida en el logro de los ODM. Para ello hace hincapié en el crecimiento económico, unido a la reducción de la pobreza, a través del aumento tanto cuantitativo como cualitativo de la AOD y el mayor acceso a sus mercados. 
podía ser en forma de créditos blandos o donaciones, pero en ningún caso podían ser Ayuda Ligada pues lo prohíben las leyes del país.

Durante estos años y hasta el 2003 son muchos los préstamos y donacio nes de países como Australia, Bélgica, Canadá, Dinamarca, Francia, Italia, Holanda, Irán, URSS, Yugoslavia. Pero, a partir de la promulgación en el año de 2003 de los "Lineamientos para la cooperación al desarrollo con socios bilaterales", el Gobierno de la India sólo acepta ayuda bilateral de los países que conforman el G-8, como son: Japón, Alemania, Gran Bretaña, Italia, Fran cia, Estados Unidos, Canadá y la Federación Rusa. Se incluye en este grupo también a la Unión Europea. Para aquellos países de la UE que no conforman el G-8 está prevista la aceptación de la ayuda siempre y cuando supere un compromiso mínimo anual de 25 millones de US\$.

Todos los demás países, incluyendo los que no han sido nombrados en las líneas anteriores, serán invitados a participar como socios bilaterales, a través de la asistencia directa a: institutos autónomos, universidades, ONG, etc. Esta modalidad está regida por "The Foreign Contribution (Regulation) Act., 1976". Asimismo se insta a aquellos que tuvieran algún proyecto en vigor a continuar con su ayuda hasta el fin de los mismos. De igual modo se les aconseja continuar sus aportaciones a través de oficinas de desarrollo de la ONU. Y por último se decide no aceptar más ayuda ligada.

De ese modo es que, desde el año 2003, el nivel de ayuda bilateral irá disminuyendo notablemente.

De los países que componen el G-8, los 4 gobiernos donantes de mayor peso son:

\section{- Japón}

El JBIC (Banco de Cooperación Internacional del Japón), es el encargado de fomentar las exportaciones y las importaciones del país, además cuenta con las operaciones de cooperación económica de ultramar, a través de la cual, otorga AOD por medio de la concesión de préstamos a largo plazo e intereses bajos para la construcción de infraestructura económica en los países en desarrollo. Hoy en día es uno de los principales donantes de fondos hacia la India a través de prestamos, para el año 2003 se han comprometido 125.004 millones de $¥$, distribuidos en 8 proyectos. Los proyectos se concentran principalmente en los sectores de energía, transporte, agua y medio ambiente 
y se distribuyen regionalmente en: Delhi, Haryana, Rajasthan, Uttaranchal, Orissa, Meghalaya, Andhra Pradesh y Bengala Occidental.

Los ODM sobre los que trabaja el JBIC son básicamente dos: erradicar la pobreza extrema y el hambre y garantizar la sostenibilidad del medio ambiente.

\section{- Reino Unido}

El DFID (Departamento de Desarrollo Internacional), es el encargado de gestionar toda la política de ayuda a los países más pobres por parte del Reino Unido, que tiene como finalidad erradicar la pobreza extrema en el mundo. El DFID viene trabajando ya desde su creación en 1997 con la India, quien actualmente representa el mayor receptor de su ayuda bilateral. Aún cuando poseen un Plan Nacional, en su mayor parte los fondos son destinados a niveles de gobierno estatales y estarán destinados a los Estados de: Andhra Pradesh, Madhya Pradesh, Orissa y Bengala Occidental. El objetivo general de la intervención se fundamenta en su contribución con el 10 Plan Quinquenal, y se compone de tres objetivos específicos: a) Elaborar un acercamiento más integrado a la lucha contra la pobreza en los estados seleccionados,b) Mejorar la infraestructura para un crecimiento más equitativo y sustentable y c) Ampliar el acceso de los pobres a servicios de mejor calidad.

Para lograr los objetivos señalados anteriormente, el gobierno del Reino Unido a través de DFID, tiene presupuestado gastar aproximadamente 750 millones de § hasta el año 2006.

El ODM al que contribuye el DFID es principalmente el de erradicar la pobreza extrema.

\section{- Alemania}

La ayuda al desarrollo bilateral del gobierno alemán se inició en 1958 y según cifras del 2003 se encuentra en la tercera posición del ranking de socios bilaterales después de Japón y UK. La agencia alemana que se ocupa de la cooperación es la GTZ. La cooperación se destina a los siguientes sectores acordados de forma conjunta con el Gobierno de la India, en diciembre de 2002: a) Política medioambiental, protección y uso sostenible de los recursos naturales, $b$ ) Energía,c) Reformas Económicas (financieras y sector privado), y d) Salud, planificación familiar y VIH / SIDA. 
Madhya Pradesh, Himachal Pradesh, Rajastán, Karnataka, Maharashtra,-Benga la Occidental y Orissa, constituyen los estados focales para la ayuda alemana.

De esta forma, desde la GTZ se apoya la consecución de los siguientes ODM: erradicar la pobreza extrema y el hambre y garantizar la sostenibilidad del medio ambiente y los objetivos relacionados con salud (4, 5 y 6$)$.

\section{- Estados Unidos ${ }^{7}$}

La USAID (Agencia de Los Estados Unidos de Cooperación), viene cola borando con la India desde hace más de 50 años, con lo cual las relaciones entre ellos son cada vez más fuertes, hasta tal punto que existe una oficina muy activa en la embajada de este país en Nueva Delhi, por medio de la cual, ha establecido una serie de acuerdos con diferentes instituciones y organizaciones involucradas en temas de desarrollo y muy en especial con el Gobierno de la India.

En este sentido, la USAID ha establecido una serie de objetivos para los cuales concentrará su estrategia: 1) Crecimiento económico, 2) Salud, 3) Preparación y respuesta contra desastres, 4) Energía y agua y 5) Oportunidades para la población más vulnerable. Además de estos cinco objetivos de manera transversal se hará énfasis en: a) Gobernabilidad, b) Género, c) Problemas urbanos, d) Asociaciones y e) Tecnología punta. Para cumplir estos objetivos se estima que la agencia utilizará para los próximos 5 años de 930 millones de US\$ a 1.160 millones de US\$.

La ayuda se localizará en los siguientes Estados: Uttar Pradesh, Jharkhand, Rajasthan, Andhra Pradesh, Karnataka y Uttaranchal.

Los ODM beneficiados, de acuerdo a los criterios establecido por USAID, serán: erradicar la pobreza extrema y el hambre, promover la igualdad en tre los géneros y la autonomía de la mujer y garantizar la sostenibilidad del medio ambiente.

\subsection{Cooperación privada}

La cooperación privada se caracteriza principalmente por trabajar entre las comunidades rurales con socios locales. Los Organismos No Guberna -

7 En diciembre 2006 un acuerdo de cooperación nuclear fue firmado entre EE.UU. y la India, aprobando el envío de combustible nuclear civil al país. 
mentales de Desarrollo (ONGD) son los actores encargados de implementar los proyectos de desarrollo, a través de la recepción de fondos destinados a ello ${ }^{8}$.

Las ONGD ofrecen intervenciones claramente enfocadas a mejorar las condiciones de vida de la población más desfavorecida en las diferentes lo calidades del país donde trabajan. En casi todos los casos los socios locales tienen un alto nivel de protagonismo y las directrices de los proyectos incor poran elementos muy afines a los ODM: pobreza, salud, educación, mujer, etc. Por lo que se podría afirmar, que, si bien su impacto es relativamente pequeño, contribuyen de forma directa al logro de la mayoría de los ODM, sobre todo a aquellos de carácter más social. Hay que tener en cuenta a la hora de valorar el trabajo de estas organizaciones, el dato de que la India ocupa el puesto 127 en el IDH elaborado por la ONU.

De esta forma, desde el punto de vista teórico, los proyectos de desarrollo que llevan a cabo, se encuadran muy bien en el marco del desarrollo humano. Es evidente que en estos proyectos se prioriza el esfuerzo para equilibrar el aspecto del desarrollo económico con los demás aspectos del desarrollo que se contemplan desde este modelo. El desarrollo es considerado en el ámbito de la cooperación de las ONGD como creación de una serie de oportunidades para la población, que le permitan, no sólo, una salida a la situación de pobreza, sino también, un desarrollo social, económico y político.

Desde un punto de vista práctico, se debe tomar en consideración la gran variedad de proyectos de desarrollo de las ONGDs: dirigidos a diferentes colectivos, sectores específicos y de desarrollo institucional. Sin embargo, debido a su escasa capacidad monetaria y técnica, sus proyectos tienen en su mayoría, una función paliativa, teniendo escaso impacto en el desarrollo global.

Su verdadero potencial se desprende de su capacidad de análisis y comprensión de las situaciones desde la óptica de la sociedad civil y su libertad para generar informes que conllevan denuncias de los abusos producidos por el sistema dominante.

8 Dada la gran cantidad de ONGD externas que trabajan en la India y las numerosas ONG loca les, se nos hace imposible un análisis sintético de cada una de ellas. Para conocer algunas de estas ONGD se señalan dos direcciones de páginas web: para ONGD españolas actuantes en la India consultarwww.indiga.org; para ONG indias consultar http://planningcommission. org.in 
Las actuaciones de las ONGD extranjeras se regulan a través de los "Li neamientos para la cooperación al desarrollo con los socios bilaterales", del departamento de asuntos económicos del ministerio de finanzas. Uno de los reglamentos fundamentales dice que la ejecución de toda ayuda se condiciona a través de las ONGD locales.

\section{Balance de la situación}

A la vista de lo señalado hasta el momento, tanto la política nacional de desarrollo como la ayuda internacional: multilateral, bilateral o privada, por lo general, están enfocadas en la práctica a la consecución de los ODM. Se concretará, en los siguientes párrafos, el avance en el logro de los diferentes objetivos. Aún cuando la revisión de dicho avance pueda ser interpretada como una evaluación de las actuaciones señaladas, hay que ser conscientes de las limitaciones que entraña la falta de datos suficientes, así como la nece sidad de profundizar en la aplicación específica de cada una de las diversas políticas concretas, lo que desborda el carácter y extensión de este artículo. Para ello se tomarán los datos de los distintos indicadores establecidos para el análisis de los ODM en dos puntos temporales, el primero será el año 1990, o en el caso de no obtenerse, los años más próximos, establecido éste como punto de referencia por la ONU; y el segundo, los datos más actuales existen tes. La base de datos escogida, principalmente, es la ofrecida por el reciente programa elaborado por el BM "World Development Indicators 2005".

Objetivo 1. Erradicar la pobreza extrema y el hambre. La incidencia de la pobreza ha disminuido en los últimos años, pasando de un 36\% en 1994 a un $29 \%$ en 2000, según el umbral de pobreza establecido nacionalmente; o de un $42 \%$ en 1993 a un 35\% en 1999, según la línea de pobreza extrema establecida por la ONU (menos de $1 \$$ al día). Aunque en números absolutos el porcentaje de personas pobres es todavía alarmante, los datos relativos hacen prever que la meta de reducir a la mitad la población pobre según el concepto de la ONU, tiene muchas posibilidades de ser alcanzada. En cuanto a la meta de reducir a la mitad la gente que sufre hambre, la proporción de personas desnutridas en el total de la población cayó desde 25\% en 1990-1992 a 21\% en 2001, siendo para el grupo de menores de 5 años, un 64\% en 1990 y un 47\% en 1999, según peso/edad. El leve descenso en el total de población y el alto porcentaje todavía prevalente en el grupo de menores, indica la necesidad de aumentar y sostener los programas de seguridad alimentaria, si se quiere conseguir dicha meta. 
Objetivo 2. Lograr la enseñanza primaria universal. La tasa bruta de matriculación en la enseñanza primaria es una de las que mejores resultados presenta; sin embargo, según el BM, ya en 1990 se daba el 99\% de matricula ciones que se da en el reciente 2003, por lo que no se podría considerar un logro de la AOD en la consecución de la meta, ya que se daba con anteriori dad. En cuanto al porcentaje que completa la primaria, se ha aumentado de $78 \%$ en 1994 a $81 \%$ en 2002, sobre el grupo de edad pertinente. No obstante, según el BAD, en 2000-2001 el porcentaje de alumnos que comenzaron el primer grado y llegaron a quinto fue de 59\%. Por último, la tasa de alfabetización muestra para el grupo de personas entre 15 y 24 años, un aumento desde un 64\% en 1990 a un 73\% en 2001, este último dato según el BAD. Lo primero en señalar sería la contradicción en los datos observados, ya que el porcentaje de finalización de la primaria es muy distinto según el BM y el BAD. De cualquier forma, dadas las circunstancias, se debería dedicar mayor esfuerzo a la calidad de la enseñanza, y no tanto a insertar alumnos en el sistema educativo.

Objetivo 3. Promover la igualdad entre los géneros y la autonomía de la mujer La consecución de la paridad entre los géneros en la enseñanza primaria y secundaria, está siendo alcanzada. Para primaria, la diferencia en 1990 entre géneros era de 26 puntos porcentuales, mientras que en 2001 era de 16. Para secundaria, la diferencia en 1990 era de 22 puntos porcentuales, siendo en 2001 de 15. Sin embargo, para estudios superiores, la diferencia se mantiene, ya que en 1990 había un 4\% y un 8\%; y en 2001, 9\% y 13\%, respectivamente para mujeres y varones sobre su grupo de población, lo que representa para ambas fechas una diferencia de 4 puntos porcentuales. En cuanto al alfabetismo femenino, los porcentajes sobre la población de mujeres con edad comprendida entre 15 y 24, para 1990 es de 54\%, y para 2000 de 65\%, siendo para los varones de $73 \%$ y $80 \%$. De esta forma, aunque sigue siendo mayor el grupo de mujeres analfabetas, se reduce la distancia entre géneros en 6 puntos porcentuales. Por otro lado, la igualdad de géneros en el acceso a los servicios básicos sigue siendo bastante baja. Según el "Informe Desarrollo Humano 2005" elaborado por el PNUD, la tasa de mortalidad de niñas entre uno y cinco años es superior en $50 \%$ a la de los niños. Al igual que el escaso peso de la mujer en la toma de decisiones como es su representación parla mentaria: en 19905 asientos eran ocupados por mujeres, aumentando a tan sólo 9 en 2004. Según el BAD, los índices de desarrollo humano relacionados al género mejoraron de 0,410 en 1992 a 0,572 en 2002. Todo ello significa que la igualdad de género no ha tenido una mejora considerable. 
Objetivo 4. Reducir la mortalidad de los niños menores de 5 añosEl indicador de mortalidad sobre el grupo de edad de menores de 5 años, ha pasado de presentar un $12 \%$ en 1990 a $8 \%$ en 2003. Aunque, se están manteniendo programas hacia el logro del objetivo, como son los implantados por UNICEF para la vacunación de la polio a toda la población infantil, la prevalencia de distintas enfermedades sobre este sector de población merma la consecu ción de la meta de reducir en $2 / 3$ partes dicha mortalidad. Según el "Informe Desarrollo Humano 2005", la India redujo su ritmo en la disminución de la mortalidad infantil a partir de 1990, época que coincide con su crecimiento económico, el argumento posible es que una vez cubiertos los servicios de salud pública básicos, el problema queda concentrado en las poblaciones más difíciles de alcanzar y a su vez más vulnerables, lo cual hace subir los costos marginales de la sanidad y modera los avances.

Objetivo 5. Mejorar la salud materna. Según el BAD, la tasa de mortalidad materna (por cada 100.000 nacimientos) disminuyo de 437 en 1991 a 407 en 1998 y el porcentaje de partos con asistencia de personal sanitario especia lizado mejoró de 34\% en 1992-1993 a 43\% en promedio entre 1995-2002. No obstante, no son resultados suficientemente buenos como para afirmar la consecución de la meta de reducir la mortalidad materna en tres cuartas partes. Para ello, debería haber una mayor eficacia en los proyectos para hacer el embarazo más seguro y mejorar el acceso de comunidades aisladas a los servicios de salud, así como también una mayor inversión por parte del gobierno en salud pública.

Objetivo 6. Combatir el VIH/SIDA, malaria y otras enfermeda\&ún el BAD, el número de personas aproximadamente que vivían con HIV/SIDA aumentó de 3,5 millones en 1998 a 4,5 millones en 2002. La prevalencia de SIDA en adultos de (15 a 24 años) es de $0,8 \%$. La tasa de protección (uso de preservativos) en las parejas era 44\% en 1991 y 48\% en 1998-1999. Según esta tendencia, muy posiblemente, no se conseguirá la meta de haber detenido y comenzado a reducir, para el año 2015, la propagación del VIH/SIDA. El PNUD, BM y CE de forma descentralizada, son los únicos organismos que dedican su ayuda al cumplimiento de dicha meta, por lo que habría que concienciar a los agentes de la cooperación bilateral de su importancia. En cuanto a la segunda meta del objetivo: (haber detenido y comenzado a reducir, para el año 2015, la incidencia de la malaria y otras enfermedades graves), los datos ofrecidos por el BAD afir man que el total de casos de malaria era de 260 por cada 100.000 habitantes en 1991, siendo de 193 en el año 2000 y el total de casos de tuberculosis disminuyó de 137 en 1996 a 123 casos por cada 100.000 habitantes en 1999. 
Objetivo 7. Garantizar la sostenibilidad del medio ambiente. La superficie cubierta por bosques muestra un aumento de $21 \%$ a $22 \%$ entre 1990 y 2000 . El uso de energía (equivalente en kilogramos de petróleo) por 1 dólar del producto interno bruto, aumentó de 3 US\$ a 5 US\$ en el período 1990 - 2002. Según el BAD, la relación entre zonas protegidas para mantener la diversidad biológica y la superficie total se incrementó de 4,4\% en 1989 a 4,7 en 2003. Las emisiones de dióxido de carbono per cápita aumentaron de 0,5 toneladas métricas en 1980 a 1,1 para el año 2000. En este mismo año, el consumo de clorofluorocarburos que agotan la capa de ozono fue de 5.614 (toneladas de PAO). Esto demuestra, que aunque se está caminando hacia la incorporación de los principios del desarrollo sostenible en las políticas de ayuda y en los programas nacionales, aún queda por recorrer. En cuanto a la meta de reducir a la mitad el porcentaje de personas que carezcan de acceso sostenible al agua potable y a servicios básicos de saneamiento (incluida también en este objetivo), los datos muestran un aumento del 68\% al 86\% entre los años 1990 y 2002 , sobre la población con acceso al agua, siendo esta mejora de $61 \%$ a $82 \%$ para zonas rurales y de $88 \%$ a $96 \%$ para zonas urbanas. Por lo que respecta al segundo indicador, el acceso de la población a los servicios de saneamiento, pasó de $12 \%$ a $30 \%$, siendo para el área rural de 1\% a 18\%, y en el área urbana de 43 a 58\% para el periodo comprendido entre 1990 y 2002. En cuanto a la última meta del objetivo (haber mejorado considerablemente, para el año 2020, la vida de por lo menos 100 millones de habitantes de tugurios), el 40\% de los hogares en los tugurios no tienen acceso seguro a agua potable y el $90 \%$ no tienen servicios sanitarios. El programa de mejoras ambientales en los tugurios urbanos busca crear instalaciones públicas adecuadas. En tres décadas, desde su implementación, este esquema ha beneficiado a más de 32 millones de habitantes de los tugurios.

Objetivo 8. Crear una alianza global de desarrollo. Como hemos visto anteriormente, la cooperación bilateral que se establece con la India está restringida geográficamente. Esto ha propiciado una disminución de la AOD de 464.190.000 US\$, desde el año 1990 hasta el 2003, lo que deja una importante laguna, al no haberse paliado este déficit con recursos públicos. No obstante, la ayuda está bien coordinada con los objetivos de desarrollo de la nación, y aunque represente menos del 1\% en su economía, realiza un importante y eficaz papel en el desarrollo humano de la India. En cuanto a los indicadores relacionados con el mercado, las importaciones de bienes y servicios representaban en 1990 el 9\% del PIB, mientras que en 2003 representan un 16\%; las exportaciones de bienes y servicios, en 1990, participaban 
en el PIB con un $7 \%$, aumentando a $14 \%$ en el año 2003. Por otra parte, la deuda externa ha aumentado de 83.628 millones de US\$, en 1990, a 113.467 millones de US\$, en el año 2003.

Con respecto a la incorporación de las nuevas tecnologías, especialmente aquellas relacionadas con la informática y las comunicaciones, entre la población, los datos analizados han dado los siguientes resultados: las líneas de teléfono y móviles por cada 100 habitantes, suponían en 1990 un 0,6, au mentado en 2003 a 4,6. Mientras que los ordenadores personales aumentaron de $0 \%$ en 1990 a $0,7 \%$ en 2002. Por último, los usuarios de Internet aparecen contabilizados por primera vez en 1992, pero siendo tan insignificante que aparecen como $0 \%$, pasando en 2003 a 17\%, el aumento más notable de todos los indicadores analizados. Por tanto, aunque las cifras son aún relevante mente bajas, no cabe duda que ha habido un gran progreso en el acceso, por parte de la población, a estos servicios.

En resumen, si bien se ha avanzado ciertamente en el camino trazado por la declaración del milenio, queda mucho por recorrer si se desean alcanzar los ODM dentro de los plazos señalados.

\section{Conclusiones}

Los resultados de las políticas nacionales de desarrollo y de la cooperación internacional en relación con los ODM, aun cuando parecen positivos, a juzgar por los datos considerados, ponen de relieve la existencia de importantes carencias por cubrir, lo que requiere extremar la atención y mejorar las líneas de actuación para poder conseguir las propuestas realizadas por la asamblea general de la ONU dentro de las fechas establecidas. Obviamente, cabe una responsabilidad principal al propio gobierno de la India, si bien la magnitud de los problemas continúa demandando el apoyo de la Comunidad Internacional.

Sin embargo, se puede afirmar, que la ayuda externa no juega un papel significativo en la economía de la India, pues representa menos del 1\% del PIB. No obstante, ha financiado la mayoría de los proyectos destinados a mejorar la infraestructura, educación, salud, igualdad de género, etc., algunos de ellos muy relacionados con los ODM.

Tras haber realizado un análisis de cada una de las actuaciones de los di ferentes socios de cooperación para el desarrollo de la India, hemos visto de 
manera positiva cómo cada uno de los agentes han coordinado sus políticas de ayuda a través del $10^{\circ}$ Plan Quinquenal de la Nación de la India. En este sentido, se considera que el enfoque prioritario que se le está dando a algunos estados más necesitados pudiese contrarrestar las marcadas diferencias de crecimiento que existe entre ellos, al igual que la relevancia que se le da a algunos sectores, en detrimento de otros. La otra cara de la moneda aparece cuando se le atribuye al Estado la responsabilidad del desarrollo de aquellos sectores que quedan más marginados, siendo él mismo el que decidió no priorizarlos y debido a la imposibilidad de llegar a todos los sectores a causa de la insuficiencia de recursos para el sector público.

En este sentido, la falta o ineficaz distribución de los recursos se señala como uno de los problemas fundamentales, se podría concluir entonces que la existencia de un sector público más extenso, que realice una mayor inversión en educación, sanidad, infraestructuras, etc. es una necesidad si se pretende un desarrollo social y progresivo en la India. El gasto público en educación ha aumentado en la última década, mientras que en sanidad se mantiene en menos de un 1\% del PIB, siendo una tercera parte del gasto militar, sector a donde se destina el doble de la cantidad de AOD que recibe el país, según el informe mundial del desarrollo humano del PNUD. Por tanto, es imprescindible que el Estado ponga más recursos en los servicios sociales. El crecimiento económico del país le ofrece esta oportunidad, no se ha de olvidar el extraordinario crecimiento industrial y comercial de la India que, junto con China, la está convirtiendo en una primera potencia económica mundial. Sin embargo, a pesar de ser uno de los más notorios exponentes del éxito de la globalización, no está logrando transformar la creación de riquezas y el aumento de ingresos en una reducción considerable de las enormes desigualdades sociales existentes.

Por otro lado, a tenor de los datos analizados, se puede señalar que, la mentablemente, los objetivos que se propone están muchas veces alejados de la situación real del país, dándose la paradoja de objetivos que deberían haberse cumplido para 2003 y que en ningún caso se han logrado. Por lo tanto, el problema lo encontramos, no tanto en definir unos objetivos ambi ciosos, sino en aplicar la conducta más razonable de que hasta que no sean logrados los ya marcados, no se elaboren nuevos objetivos.

Acorde con este hecho, los deseos de muchos de los donantes en cumplir con las metas señaladas, se transforman en una demanda de informes con indicadores de éxito, cuya única consecuencia es falsear una realidad que 
conlleva a su vez el riesgo de dañar la eficacia de los futuros proyectos de desarrollo implementados en la India.

En esta línea, se ha de señalar que los resultados positivos en algunas de las metas de los correspondientes ODM no proporcionan una visión certera de la situación real. Así, si bien los índices de paridad escolar están siendo alcanzados, esto no significa de manera alguna la transformación de los roles estereotipados existentes en la sociedad que responsabilizan a las mujeres del cuidado y atención de sus hijos/as, de la gestión de mejoras para su comunidad y, en un porcentaje cada vez mayor, de garantizar el sustento económico de la familia.

Además, dadas las características del país, especialmente su estructura social basada en una estratificación según la casta de procedencia, una apropiada estrategia de desarrollo sería aquella basada en la creación y movilización de grupos sociales, que actúen activamente en el desarrollo del país o de su región. Esto implica, no sólo dar prioridad a las castas más bajas en los distintos proyectos ejecutados, sino también ejecutar proyectos concretos destinados al desarrollo de sus capacidades y recursos tanto sociales y económicos, como políticos.

La cobertura educativa de la que dispone el país posibilita la transmisión y el enriquecimiento de los valores culturales y morales comunes. En esos valores asientan el individuo y la sociedad su identidad y su dignidad. La educación debe de ir más allá de completar las tasas de matriculación y el grado de supervivencia, se ha de tomar como la base para proporcionar un cambio tanto económico como social, instrumento indispensable para lograr un desarrollo humano ligado a su libertad, posibilitando así una conciencia social que permita la movilización de los estratos sociales.

Esta estrategia de desarrollo endógeno se echa de menos en el panorama de cooperación descrito previamente, la coordinación de las ayudas acorde con los objetivos nacionales, guiados por la declaración de los objetivos del milenio, deja poco lugar a la implementación de programas que no se vean contemplados bajo los 8 objetivos. De esta forma, pocos son los proyectos encaminados de forma concreta a incentivar la participación ciudadana 0 crear y mejorar la organización de las comunidades locales.

Es por ello, que los ODM se deben considerar como unos mínimos de donde partir, una base para generar un desarrollo inicialmente social más completo y equitativo, que se han de adecuar permanentemente a las características propias del país en cuestión. 


\section{Referencias}

Informes consultados

AlBARES, J. Manuel, (2005), 2005: año cero para la ayuda mundial y los Obje tivos de Desarrollo del Milenio , Madrid, Fundación para las Relaciones Internacionales y el Diálogo Exterior.

BAD, (2004), Country Strategy and Program Update 2002 - 2007 India.

BIRD, (2006), World Developemnt Indicators 2005.

Departmentof Economic Affairs, (2004),Guidelines for Development Co-operation with Bilateral Donors, New Delhi, Government of India.

Department of Economic Affairs. PMU Division, (2005), Cooperation Policy, New Delhi, Government of India.

Diario Oficial de la Unión Europea, (1994),Acuerdo de cooperación entre la Comu nidad Europea y la Republica de India para la asociación y el desarrollo.

- (2003), Sobre la eficacia de la gestión de la Comisión en la cooperación al desarrollo en la India respectodel objetivo de ayudar a los pobres y obtener efectos sostenibles, acompañado de las respuestas de la Comisión Informe Especial № 10/2003.

DIFD, (2004), DIFD's Country Plan in India 2004-2008 . Britain Development Programme in India.

Economy Ministry, (2003), Informe sobre la ayuda externa 2002 - 2003, New Delhi, Government of India.

EUROSTEP, (2003), Informe sobre la erradicación de la pobreza en el sud de Asia. Sociedad civil, gobierno nacional, Comisión Europea y Eurostep en un multipolar mundo.

European Commission, (2002), EC Country Strategy Paper India 2002- 2006.

- (2003), La cooperación Europea con América Latina y Asia: Revisando el Reglamento - Prueba de fuego para el compromiso de la Unión Europea con la erradicación de la pobreza.

- (2004), EU-India Strategic Partnership India 2002.

Ministry of FinAnce, (2004), Informe sobre la Ayuda Oficial al Desarrollo, India 2003-2004, Government of India, Asian Development Outlook. 
Ministry of Water Resources, (2002), National Water Policy, New Delhi, Government of India.

Planning Commission, (2002), Water Supply and Sanitation in India, New Delhi, Government of India.

- (2001), National Health Policy - 2002. Tenth Five Year Plan 2002-07, New Delhi, Government of India.

- (2004), Annual Report 2002-2003, New Delhi, Government of India.

- (2005), Annual Report 2003-2004, New Delhi, Government of India.

PNUD - R.anning Commission, (2002),India National Human Development Report 2001, New Delhi, Government of India.

PNUD, (2002), Country Evaluation India 2002.

PNUD, (2005), Informe de Desarrollo Humano 2005.

SACH, D. Jeffrey, (2005), 2005 - The year of development , Barbados, UN Mi llennium Project.

USAID, (2004), India Annual Report for Year 2004.

World Health Organization, (2002), Country health profile India.

\section{Páginas Web consultadas}

Directorio de páginas web del gobierno de la India, http://goidirectory.nic. in/, acceso: abril 2006.

Revista Gloobal hoy, www.gloobal.info/iepala/gloobal., acceso: marzo 2006.

Gateway Development,http://home.developmentgateway.org/DataStatistics Foundation, acceso: abril 2006.

Goverment of India. Ministry of Finance, http://finmin.nic.in/stats data/international reports india/index.html, acceso: abril 2006.

Goverment of India. Ministry of External Affairs, http://meaindia.nic.in/, acceso: abril 2006.

Goverment of India. Planning Comision, http://planningcommission.nic. in/data/ngo/ngof.htm, acceso: abril 2006.

Mesa Redonda "Managing for Development Results, $\quad$ http://www.mfdr. org/4Partnerships.html, acceso: mayo 2006. 
Revista electrónica de recursos en Internet sobre economía y ciencias socia les, http://www.ub.es/geocrit/arac-71.htm, acceso: marzo 2006.

ONG Indiga, http://www.indiga.org/bha/geo.htm, acceso: marzo 2006.

Organización para la Cooperación y el Desarrollo Económico (OCDEhttp:// www.oecd.org/dataoecd/50/17/5037721.htm, acceso: mayo 2006.

Unión Europea, http://comm/europeaid/tender/gestion/index en.htm, ac ceso: abril 2006.

Unión Europea, http://europa.eu.int/comm/europeaid/tender/gestion/in dex en.htm, acceso: abril 2006. 